\title{
Total marrow and lymphoid irradiation with helical tomotherapy: a practical implementation report
}

\author{
Srinivas Chilukuri', Sham Sundar ${ }^{1}$, Rajesh Thiyagarajan², Jose Easow ${ }^{3}$, Mayur Sawant ${ }^{2}$, Ganapathy Krishanan ${ }^{2}$, \\ Pankaj Kumar Panda ${ }^{4}$, Dayananda Sharma ${ }^{2}$, Rakesh Jalali ${ }^{1}$ \\ ${ }^{1}$ Department of Radiation Oncology, Apollo Proton Cancer Centre, Chennai, India \\ ${ }^{2}$ Department of Medical Physics, Apollo Proton Cancer Centre, Chennai, India \\ ${ }^{3}$ Department of Haematology, Blood and Marrow Transplantation, Apollo Specialty Hospital, Chennai, India \\ ${ }^{4}$ Department of Clinical Research, Apollo Proton Cancer Centre, Chennai, India
}

Received: July 25, 2020

Revised: September 9, 2020

Accepted: September 11, 2020

\section{Correspondence:}

Srinivas Chilukuri

Department of Radiation Oncology,

Apollo Proton Cancer Centre, 4/661,

Dr Vikram Sarabai Instronic Estate

7th St, Dr. Vasi Estate, Phase II,

Tharamani, Chennai, Tamil Nadu

600096, India

Tel: +91-9885233318

E-mail:drsrinivas_c@apollohospitals.com ORCID:

https://orcid.org/0000-0003-0491-7935
Purpose: To standardize the technique; evaluate resources requirements and analyze our early experience of total marrow and lymphoid irradiation (TMLI) as part of the conditioning regimen before allogenic bone marrow transplantation using helical tomotherapy.

Materials and Methods: Computed tomography (CT) scanning and treatment were performed in head first supine (HFS) and feet first supine (FFS) orientations with an overlap at mid-thigh. Patients along with the immobilization device were manually rotated by $180^{\circ}$ to change the orientation after the delivery of HFS plan. The dose at the junction was contributed by a complementary dose gradient from each of the plans. Plan was to deliver $95 \%$ of 12 Gy to $98 \%$ of clinical target volume with dose heterogeneity $<10 \%$ and pre-specified organs-at-risk dose constraints. Megavoltage-CT was used for position verification before each fraction. Patient specific quality assurance and in vivo film dosimetry to verify junction dose were performed in all patients.

Results: Treatment was delivered in two daily fractions of 2 Gy each for 3 days with at least 8-hour gap between each fraction. The target coverage goals were met in all the patients. The average person-hours per patient were 16.5, 21.5, and 25.75 for radiation oncologist, radiation therapist, and medical physicist, respectively. Average in-room time per patient was 9.25 hours with an average beam-on time of 3.32 hours for all the 6 fractions.

Conclusion: This report comprehensively describes technique and resource requirements for TMLI and would serve as a practical guide for departments keen to start this service. Despite being time and labor intensive, it can be implemented safely and robustly.

Keywords: Total body irradiation, Lymphoid irradiation, Stem cell transplantation, Hematopoietic, Helical tomotherapy, Resource allocations

\section{Introduction}

The total body irradiation (TBI) with extended source-to-skin distance (SSD) is a simple and robust technique to deliver myeloablative treatment as part of the conditioning regimen for allogeneic bone marrow transplant (ABMT) for myeloid and lymphoid leukemia. However, this modality does not spare organs-at-risk (OAR) except lungs and results in large heterogeneities in radiation dosage across the body. Total marrow irradiation (TMI) targets the entire skeleton and lymphoid tissues while sparing the organs at risk such as parotids, oral cavity, lens, thyroid, lungs, heart, bowel, kidneys, liver, breast, etc., and is emerging as an alternative to TBI as it has shown reasonable safety and efficacy in phase I/II trials [1-6]. Addition of lymphoid irradiation to TMI which amounts to total

Copyright (C) 2020 The Korean Society for Radiation Oncology

This is an Open Access article distributed under the terms of the Creative Commons Attribution Non-Commercial License (http://creativecommons.org/licenses/by-nc/4.0/) which permits unrestricted non-commercial use, distribution, and reproduction in any medium, provided the original work is properly cited. 
marrow and lymphoid irradiation (TMLI) has the potential to reduce the rejection against the lymphocytes in donor marrow. Currently several groups are evaluating its role in standard and high-risk acute leukemia [7]. TMLI also enables the possibility of dose escalation which has been shown to reduce the rate of recurrences [8] and hence is an attractive treatment option for patients at higher risk of treatment failures.

Although a few groups have published the technique and clinical results of TMLI, there is a significant variation among the published series on various aspects of immobilization, target delineation including margin recipe, treatment optimization, plan evaluation and treatment delivery. Apart from these variations, the process of TMLI itself is laborious and time intensive which makes adoption of this technique a formidable challenge.

Based on available human and technical resources at our center, we have attempted to standardize the methodology of TMLI; analyze the resource and time requirements to accomplish this; and evaluate our initial experience including dosimetry, patient specific quality assurance and patient set-up parameters using helical TomoTherapy (Accuray Inc., Sunnyvale, CA, USA) at our center.

\section{Materials and Methods}

Patients were considered for this procedure after due discussions in the multi-disciplinary tumor board in close coordination with the hemato-oncology team. Feasibility for TMLI in these patients was based on the ability and willingness to lie down on the treatment couch for at least 1.5 hours. All patients signed an informed consent document before the procedure. Our experience of treating the first five consecutive adult patients (age $>18$ years) has been used in this practical implementation report.

\section{Simulation}

Simulation was done after the insertion of all catheters or venous access as deemed fit by the hemato-oncology team. Patients were positioned supine in a whole-body vacuum bag encompassing the entire body in a neutral position and a 5-clamp thermoplastic mask on a standard neck support was made to immobilize the head and shoulder (Fig. 1A, 1B). The arm, forearm and hands were placed closely touching the lateral aspect of the body. The knees were placed in a comfortable position and the height was adjusted on the vacuum bag. In males, the scrotum was strapped to the lower abdomen and marked. The firm impression of heel and toes on the vacuum bag was captured. Before acquiring computed tomography (CT) imaging, a thin copper wire was placed over the mid-thigh bilaterally. Lines were drawn horizontally over upper and lower limbs with corresponding lines over the vacuum bag to aid reproducibility (Fig. 1C).

As helical TomoTherapy unit can treat till a maximum length of $135 \mathrm{~cm}$, two sets of CT scans (Aquilion LB; Toshiba, Tokyo, Japan) of 5-mm slice thickness in free-breathing were acquired for each patient. The first scan was acquired from vertex to distal thigh (at least $5 \mathrm{~cm}$ beyond the wire placed over mid-thigh) in the head first supine (HFS) position. Then the patient along with the immobilization was rotated by $180^{\circ}$ and a second CT scan was acquired from toes (entire vacuum bag to be included) to the upper thigh (at least $5 \mathrm{~cm}$ beyond the wire placed over mid-thigh) in feet first supine (FFS) position. Both scans encompassed the entire thigh to aid in registration for the evaluation of the summated plan. For the HFS scan the CT reference point was marked over the chin and for the FFS scan over the knee.

\section{Target delineation}

CT images were transferred to RayStation treatment planning sys-
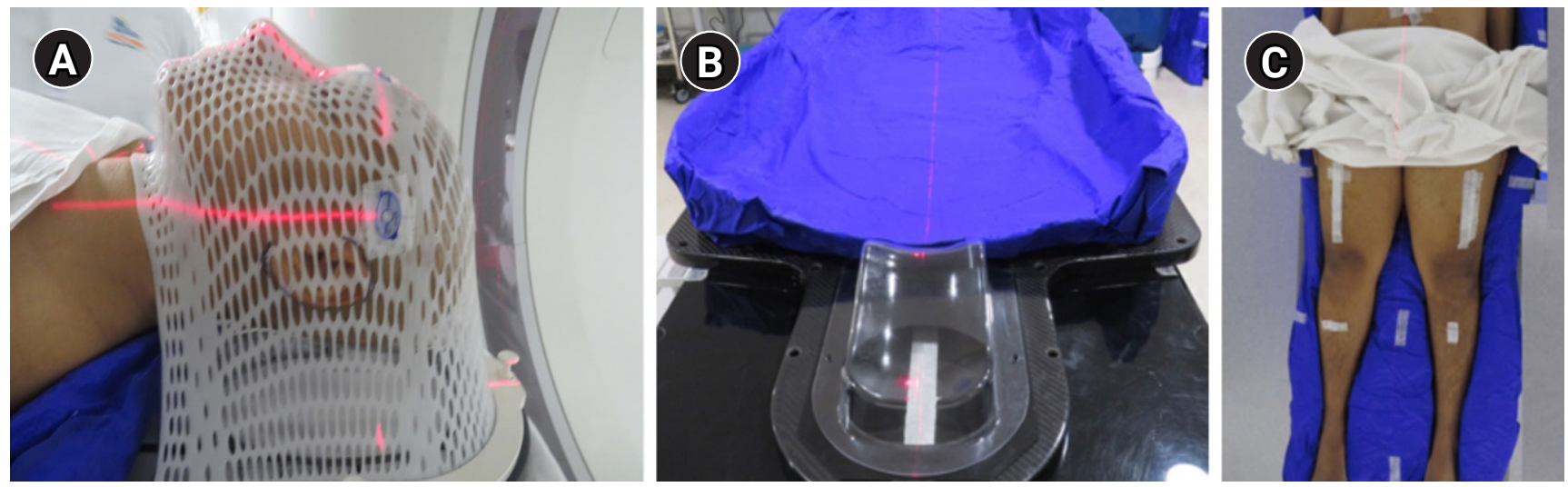

Fig. 1. (A) Image of patient immobilized in a thermoplastic mask with neck support, (B) position of the neck support and vacuum bag, (C) patient positioned in the vacuum bag with corresponding horizontal lines on the vacuum bag and over the upper and lower limbs of the patient. 
tem V7.0 (RaySearch Laboratories, Stockholm, Sweden), which is the preferred system for target delineation in our department. Clinical target volume (CTV) included entire bony skeleton, brain, testes, spleen and major lymphatics. CTV defined on HFS and FFS scan included bones auto-segmented based on grey level (Hounsfield units [HU]) thresholding ranging between 250-1700 HU and edited appropriately. Bony CTV was split into multiple segments (skull, chest, upper/lower limbs, vertebra, and pelvis) to enable differential planning target volume (PTV) margins. Mandible, hyoid bone, patella, and larynx were excluded from CTV. Isotropic PTV margin of 3 $\mathrm{mm}, 5 \mathrm{~mm}, 7 \mathrm{~mm}, 10 \mathrm{~mm}$, and $5 \mathrm{~mm}$ were given to CTV skull, vertebra, chest/spleen, upper limb (and lower limb for FFS scan), and pelvis (and scrotum), respectively. For patients with maximum lateral separation exceeding $45 \mathrm{~cm}$, an additional margin was created for PTV upper limbs since the field-of-view of megavoltage CT (MVCT) was limited to $40 \mathrm{~cm}$.

Lymphatics delineated included cervical, supraclavicular, mediastinal, axillary, entire para-aortic chain, external and internal iliac, and inguinal nodes (Fig. 2). A uniform PTV margin of $5 \mathrm{~mm}$ was applied to generate lymph nodal PTV. Individual PTV's generated were

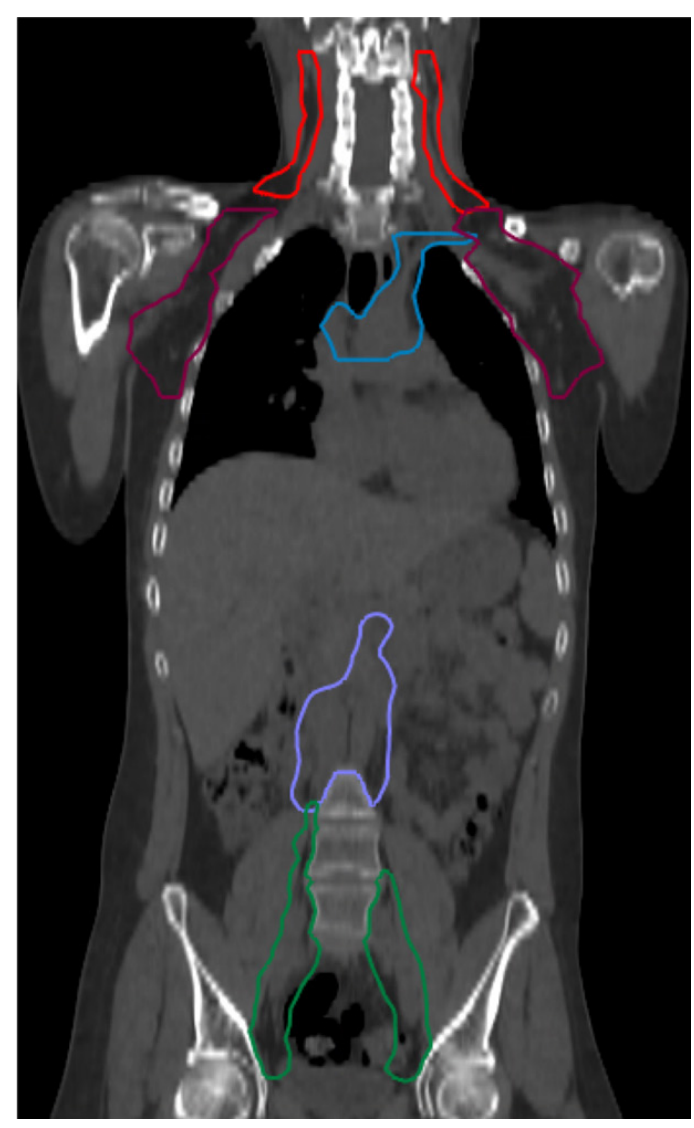

Fig. 2. Delineated lymphatic chains (cervical, red color; axillary, purple; cobalt blue, mediastinal up to hilar; light cobalt blue, para-aortic lymph node; pelvic lymph nodal chains, green). summated to create PTV. OAR delineated were eyes, lens, midline mucosa (oral, pharyngeal, laryngeal, tracheal and esophageal mucosa), lungs, heart, liver, bowel, kidneys, parotids, thyroid, breast, and ovaries. The primary planning goals were to ensure 98\% of CTV to receive $11.4 \mathrm{~Gy}$ ( $95 \%$ of $12 \mathrm{~Gy}$ ) with $\mathrm{D}_{2 \%}$ of CTV not exceeding 13.2 Gy ( $110 \%$ of 12 Gy), Lung $D_{50}$ not exceeding 8.5 Gy and no volume of OAR receiving > 12.6 Gy (105\% of 12 Gy).

\section{Treatment planning, plan evaluation and patient- specific quality assurance (PSOA)}

We used the Precision planning system (iDMS version 1.1.1.1; Accuray Inc.) for optimization and dose calculation purposes for treatment delivery on Radixact X9 tomotherapy system (Accuray Inc.). For optimization, the entire target volume in HFS and FFS images series was divided into three parts, namely PTV-upper, PTVlower, and PTV-Junction. The length of the junction was $10 \mathrm{~cm}$ (between the upper thigh and the knee) and it was divided into five sub-volumes in each CT image series to create a dose gradient. They were labeled as PhyUG2Gy, PhyUG4Gy, PhyUG6Gy, PhyUG8Gy, PhyUG10Gy, PhyLG2Gy, PhyLG4Gy, PhyLG6Gy, PhyLG8Gy, and PhyLG10Gy. The copper wires placed on the left and right mid-thigh were used as common reference markers to correlate the axial positions from both HFS and FFS CT image series. Using this common reference marker, the extension and location of the three PTV's were verified in both the CT image series.

As a standard protocol CT tabletop in the image series was replaced by the Radixact couch model. The patient position was set using a green laser in such a way that the entire body was fit within the field of view. Two separate treatment plans were generated on each of the scans (HFS and FFS) to cover the entire target with a complementary dose gradient across the junction (Fig. 3A, 3B).

Plan setup parameters were chosen to meet the clinical goals of the target. Pitch from 0.3 to 0.43 , field width of $5 \mathrm{~cm}$, modulation factor of 2.5-3.5 for HFS orientation and 2.15-2.5 for FFS were used. A higher heterogeneity was accepted near the upper limbs especially near the forearm and hands. Pitch values were optimized to have minimal thread effect and good target coverage. Higher modulation factors were used for initial iterations and subsequently modified to have efficient beam delivery time.

For summation of the plans generated on HFS and FFS CT's, RayStation treatment planning system (TPS) was used due to limitation in summating plans generated on two differently referenced CT's on Precision TPS. Deformable image registration (DIR) of both these CT's were performed using anatomically constrained deformation algorithm (ANACONDA) on RayStation, manually verified and edited if required. Using the anatomical map from DIR the dose on the FFS CT series was deformed onto the HFS CT series. The dose grids 

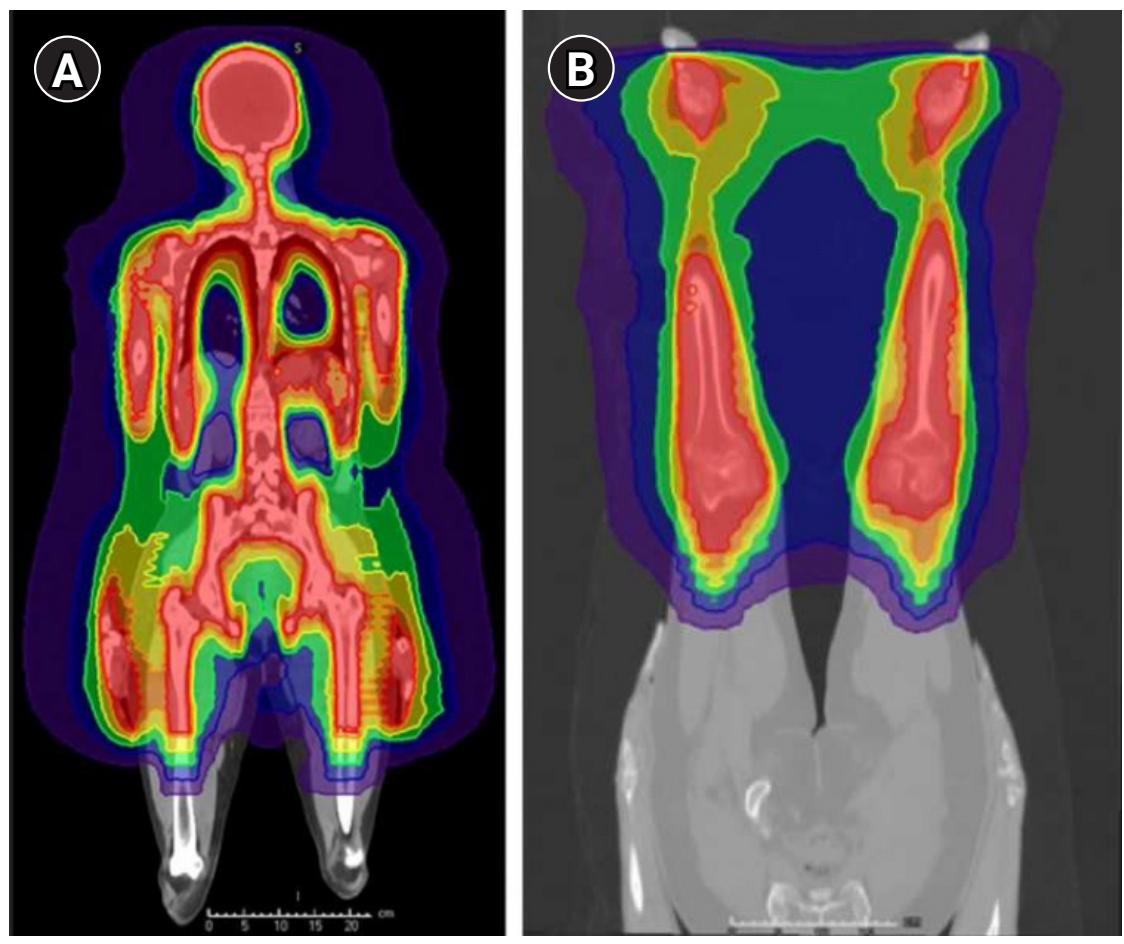

Fig. 3. (A) Head first supine plan and (B) feet first supine plan.
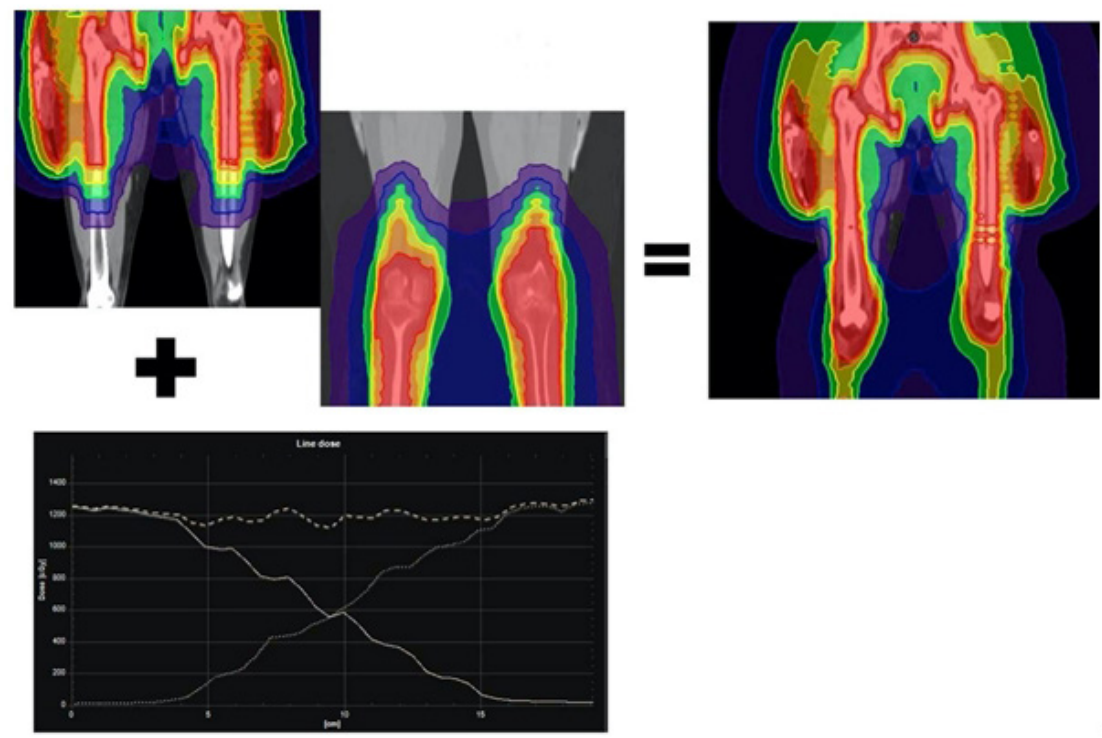

Fig. 4. Combined plan at the junction and the dose profile across the junction after combining head first supine and feet first supine plans.

from the each of the plans were summated on HFS CT series and the dose profile across the junction (Fig. 4) was evaluated.

As part of the PSOA, we measured the dose using a cylindrical cheese phantom (Accuray Inc.) with the A1SL Ion Chamber (Standard Imaging Inc., Middleton, WI, USA) and dose map using ArcCHECK helical diode array detector (Sun Nuclear, Melbourne, FL, USA). Since the target volume exceeded $120 \mathrm{~cm}$, multiple point doses ( 3 for HFS plan and 2 for FFS plan) were measured to cover the entire target. Each point dose measurement was associated with a specific quality assurance plan. Before each measurement phantom position was verified using MVCT image registration with planning CT. The measured dose maps were compared with the dose from TPS and evaluated using gamma criteria of 3\% dose difference and $3 \mathrm{~mm}$ distance to agreement. The mean gamma pass- 
ing rate was 97.82\% (96.4\%-99\%) which was above our acceptance limit ( $\geq 95 \%)$.

\section{Treatment delivery and in vivo dosimetry}

Pre-treatment imaging and treatment delivery were divided as the patient was treated in both HFS and FFS positions as described in the pictogram (Fig. 5). Treatment was interrupted each time after a pre-specified time for image verification. In HFS treatment, the first MVCT scan was obtained from lower neck to upper abdomen level to correct any significant longitudinal and yaw errors manually. The

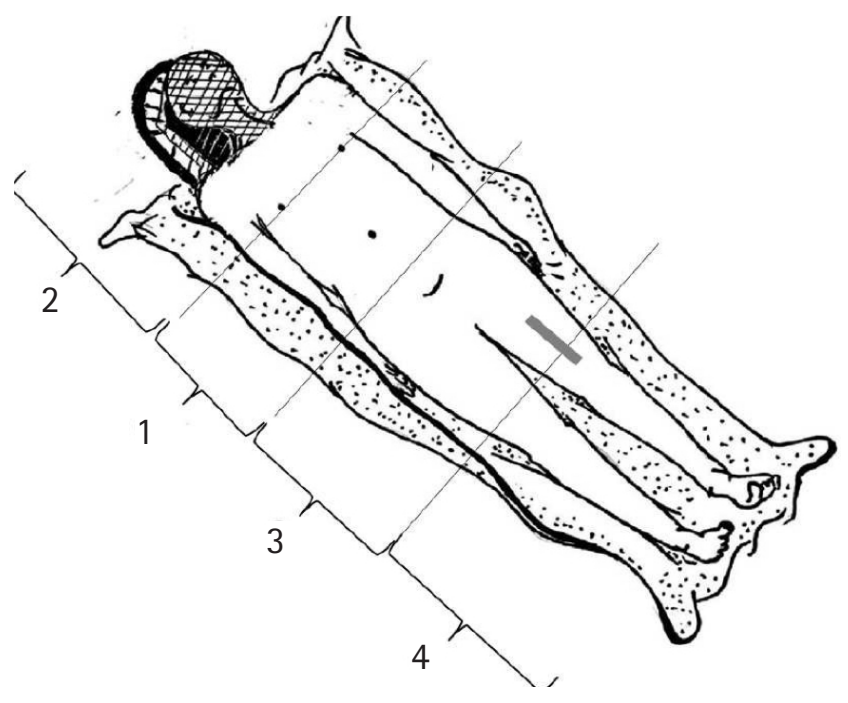

Fig. 5. The sequence of pre-treatment imaging and the position of the film on thigh. second MVCT was acquired from the vertex to the mid-chest level. After applying the necessary lateral and vertical corrections, patient was treated up to the upper abdomen. The third MVCT scan was obtained encompassing the entire abdomen up to mid-thigh, the position of scrotum was verified, and the rest of the patient treatment was completed for HFS plan. Subsequently, the patient was rotated by $180^{\circ}$ (in yaw plane) with the same immobilization and alignment in place for FFS treatment plan. The fourth MVCT was acquired from ankle to thigh, couch corrections were applied (including the longitudinal corrections since the patient was moved manually) and treatment was delivered with FFS plan. After couch corrections, before the delivery of treatment, dose-wash from the treatment plan were over-laid and manually verified for PTV to be covered by $95 \%$ dose-wash. The dosimetric accuracy of the junction was verified using GafChromic EBT3 film placed over the junction.

\section{Results}

Five patients ( 3 males and 2 females) with a median age of 36 years (range, 25 to 49 years) underwent this procedure from the 7th-5th day before ABMT. The median patient height was $167 \mathrm{~cm}$ (range, 160 to $178 \mathrm{~cm}$ ). All patients received 2 daily fractions of 2 Gy each for 3 days with a minimum gap of 8 hours between each fraction. The mean beam-on-time per fraction was 33.25 minutes with a dose rate of $1051 \mathrm{MU} /$ minute. The resources and time requirements for the treatment planning and delivery procedure have been summarized in Table 1.

The desired target coverage and OAR sparing was satisfactorily

Table 1. Time, resource requirements for the TMLI (as per local practice)

\begin{tabular}{|c|c|c|}
\hline & Median time ( $\mathrm{hr}$ ) & Resource \\
\hline \multicolumn{3}{|l|}{ Process } \\
\hline Simulation and image acquisition & 1.50 & $\begin{array}{l}2 \text { Radiation therapists (verified by medical phys- } \\
\text { icist and radiation oncologist) }\end{array}$ \\
\hline Target delineation & 6 & 1 Radiation oncologist \\
\hline \multirow{2}{*}{ Optimization and dose calculation } & \multirow{2}{*}{$\begin{array}{l}44 \text { (effective working hours spent by } \\
\text { physicist }=12 \text { ) }\end{array}$} & Medical physicist \\
\hline & & $\begin{array}{l}\text { 1-hour verification by second medical physicist } \\
\text { (partially shared time with other activities) }\end{array}$ \\
\hline Plan evaluation & 1 & Medical physicist and radiation oncologist \\
\hline Patient specific quality assurance & 1.50 & 2 Medical physicists \\
\hline Treatment delivery fraction_1 & 1.75 & $\begin{array}{l}2 \text { Radiation therapists (verified by medical phys- } \\
\text { icist and radiation oncologist) }\end{array}$ \\
\hline $\begin{array}{l}\text { Treatment delivery subsequent fractions (5\#) and } \\
\text { in vivo dosimetry }\end{array}$ & 1.50 & $\begin{array}{l}2 \text { Radiation therapists (verified by medical phys- } \\
\text { icist and radiation oncologist) }\end{array}$ \\
\hline \multicolumn{3}{|l|}{ Summary of person hours } \\
\hline Therapist & 21.50 & - \\
\hline Medical physicist & 26.75 & - \\
\hline Radiation oncologist & 16.25 & - \\
\hline In-room time for all fractions & 9.25 per patient ${ }^{\mathrm{a})}$ & - \\
\hline
\end{tabular}

TMLI, total marrow and lymphoid irradiation.

a) This excludes the treatment room preparation time which is about 15 minutes before each fraction. 
achieved in all patients. The dose-volume parameters achieved have been summarized in Table 2. The dose homogeneity was acceptable with a mean homogeneity index (based on the International Commission on Radiation Units \& Measurements [ICRU] Report 83) of 0.170 (range 0.147 to 0.190 ) for the combined PTV (PTV-upper + PTV-lower + PTV-junction). A higher homogeneity index (a higher inhomogeneity) was accepted only for PTV upper limbs (mean $\mathrm{HI}=0.327$, range 0.197 to 0.441). The mean of lung $D_{50}$, kidney $D_{50}$, heart $D_{50}$, liver $D_{50}$, and max dose to mucosal structures achieved were 8.15 Gy, 7.5 Gy, 8.17 Gy, 7.41 Gy, and $12.48 \mathrm{~Gy}$, respectively. The liver was included in the target volume for the patient \#2, which led to a higher lung $D_{50}$. For the calculation of average $D_{50}$ for liver, this patient was excluded (Fig. 6).

Setup errors were minimal in all patients based on MVCT verification (Table 3). After manually adjusting patient position (if required) based on the first MVCT at the thoraco-abdominal level and applying the necessary couch corrections in the head neck re- gion after the second MVCT, the subsequent imaging did not necessitate further corrections in the longitudinal axis.

The PSOA results achieved for all the patients were satisfactory as per our institutional thresholds and have been summarized in Fig. 7. On qualitative comparison of TPS calculated dose profile and measured dose profile (in vivo film dosimetry) at the same level no abnormal spikes in dose were observed in all the patients (within our threshold of $+10 \%$ ) (Fig. 8). All patients were successfully engrafted and are in remission at a median follow-up of 7 months

Table 3. Results of setup corrections (unit: $\mathrm{mm}$ )

\begin{tabular}{lccc}
\hline Site & Lateral $(\mathrm{x})$ & Longitudinal $(\mathrm{y})$ & Vertical $(\mathrm{z})$ \\
\hline Head and neck & $-0.1 \pm 4$ & $-2.0 \pm 3$ & $-0.7 \pm 3$ \\
Thorax & $0.1 \pm 4$ & 0 & $1.0 \pm 3$ \\
Abdomen & $1.0 \pm 4$ & 0 & $2.0 \pm 3$ \\
Legs & $0.1 \pm 4$ & $-0.4 \pm 2$ & $-0.1 \pm 2$ \\
\hline
\end{tabular}

Values are presented as mean \pm standard deviation.

Table 2. Dose volume parameters achieved (unit: Gy)

\begin{tabular}{|c|c|c|c|c|c|c|c|c|c|c|c|c|c|}
\hline & \multicolumn{10}{|c|}{ Clinical target volume } & \multicolumn{3}{|c|}{ Planning target volume } \\
\hline & \multicolumn{2}{|c|}{ Skull } & \multicolumn{2}{|c|}{ Chest } & \multicolumn{2}{|c|}{ Trunk } & \multicolumn{2}{|c|}{ Upper limb } & \multicolumn{2}{|c|}{ Lower limb } & \multirow{2}{*}{$\begin{array}{c}\text { Upper } \\
D_{95}\end{array}$} & \multirow{2}{*}{$\begin{array}{c}\text { Junction } \\
D_{95}\end{array}$} & \multirow{2}{*}{$\begin{array}{c}\text { Lower } \\
D_{95}\end{array}$} \\
\hline & $\mathrm{D}_{98}$ & $\mathrm{D}_{2}$ & $\mathrm{D}_{98}$ & $\mathrm{D}_{2}$ & $\mathrm{D}_{98}$ & $\mathrm{D}_{2}$ & $\mathrm{D}_{98}$ & $\mathrm{D}_{2}$ & $\mathrm{D}_{98}$ & $\mathrm{D}_{2}$ & & & \\
\hline Patient \# 1 & 12.0 & 12.6 & 12.0 & 12.6 & 11.8 & 13.0 & 12.0 & 13.8 & 11.8 & 12.6 & 11.8 & 11.2 & 11.9 \\
\hline Patient \#2 & 11.8 & 12.9 & 11.9 & 12.7 & 11.7 & 12.9 & 10.8 & 14.0 & 11.8 & 13.0 & 11.7 & 11.4 & 12.0 \\
\hline Patient \#3 & 11.6 & 13.0 & 12.2 & 12.8 & 12.1 & 13.7 & 10.7 & 14.0 & 11.7 & 12.7 & 11.1 & 11.1 & 11.9 \\
\hline Patient \#4 & 11.9 & 12.7 & 11.8 & 12.7 & 11.8 & 12.7 & 11.5 & 12.9 & 11.9 & 13.0 & 11.7 & 11.4 & 11.9 \\
\hline Patient \#5 & 11.9 & 12.6 & 12.0 & 12.6 & 12.0 & 12.7 & 11.5 & 13.1 & 11.5 & 12.7 & 11.8 & 11.4 & 11.9 \\
\hline Mean \pm SD & $\begin{array}{c}11.8 \pm \\
0.1\end{array}$ & $\begin{array}{c}12.7 \pm \\
0.2\end{array}$ & $\begin{array}{c}11.9 \pm \\
0.2\end{array}$ & $\begin{array}{c}12.7 \pm \\
0.1\end{array}$ & $\begin{array}{c}11.9 \pm \\
0.2\end{array}$ & $\begin{array}{c}13.0 \pm \\
0.4\end{array}$ & $\begin{array}{c}11.3 \pm \\
0.5\end{array}$ & $\begin{array}{c}13.5 \pm \\
0.5\end{array}$ & $\begin{array}{c}11.7 \pm \\
0.2\end{array}$ & $\begin{array}{c}12.8 \pm \\
0.2\end{array}$ & $\begin{array}{c}11.6 \pm \\
0.3\end{array}$ & $\begin{array}{c}11.3 \pm \\
0.2\end{array}$ & $\begin{array}{c}12.0 \pm \\
0.1\end{array}$ \\
\hline
\end{tabular}

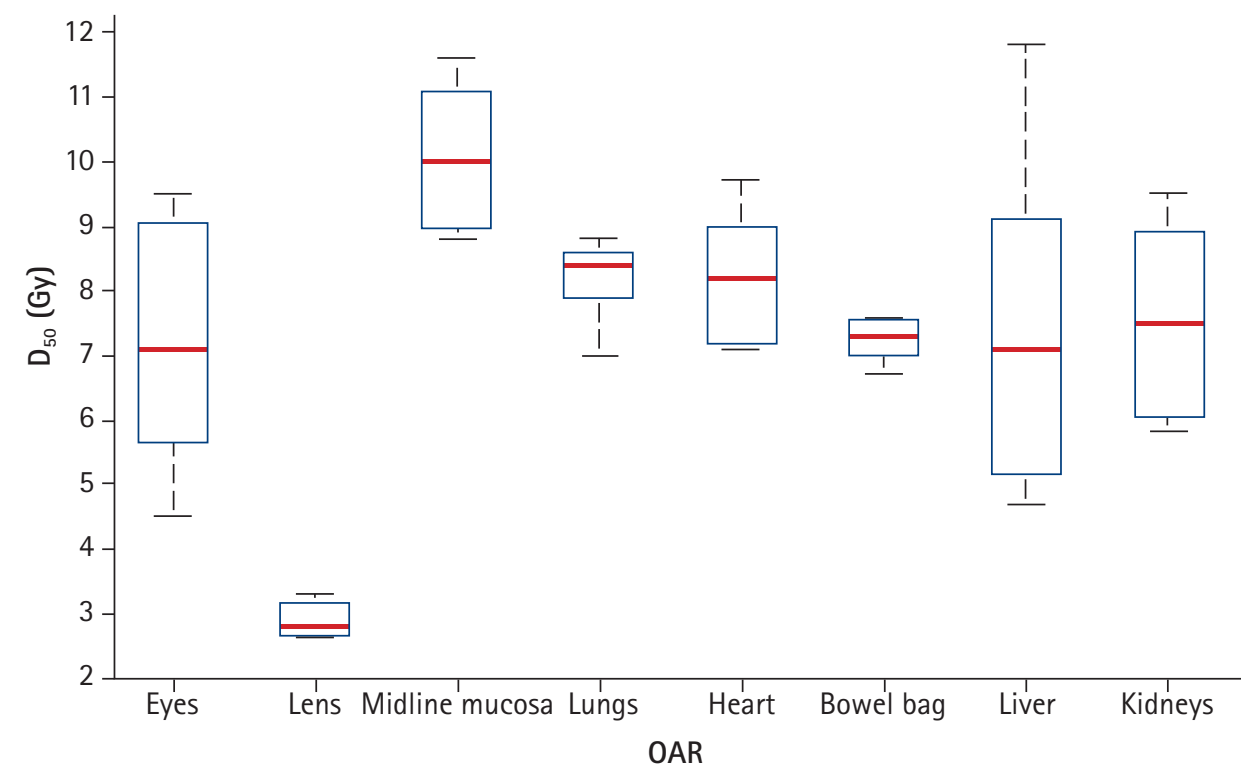

Fig. 6. Box plot of $D_{50}$ and organs-at-risk $(O A R)$ of 5 patients. Bars indicate median and interquartile range. 


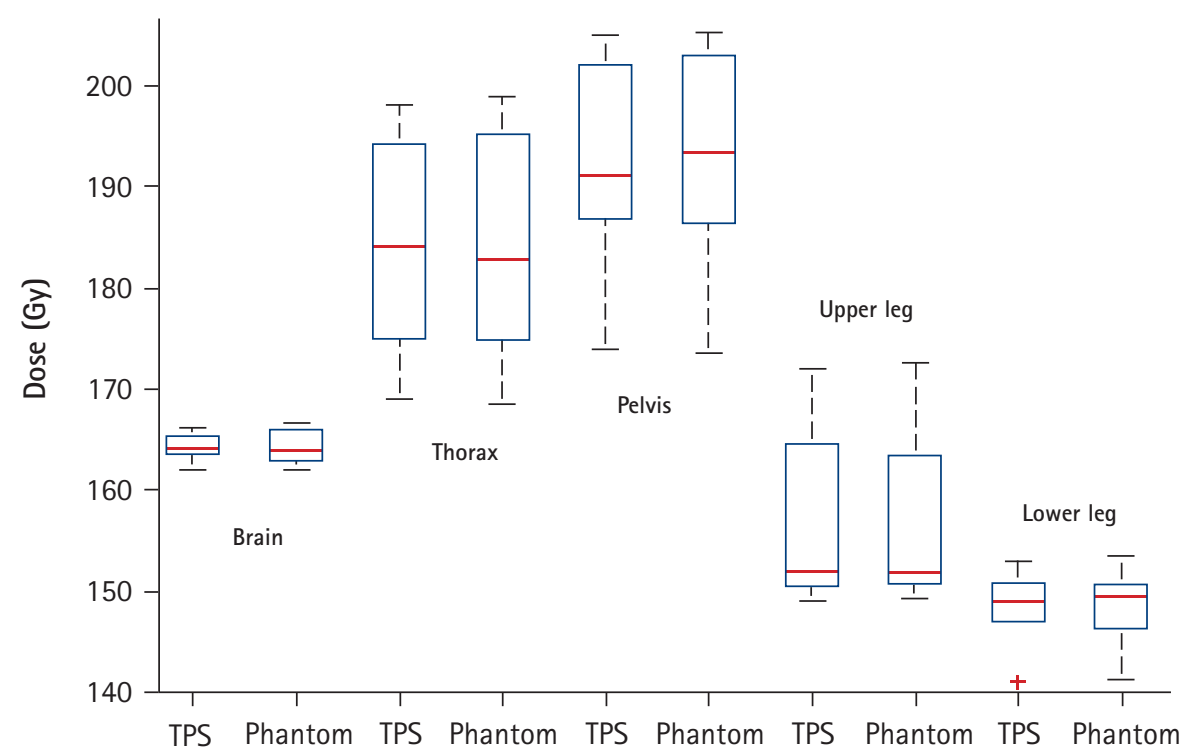

Fig. 7. Box plot of patient-specific quality assurance results measured by the treatment planning system (TPS) and the Phantom.
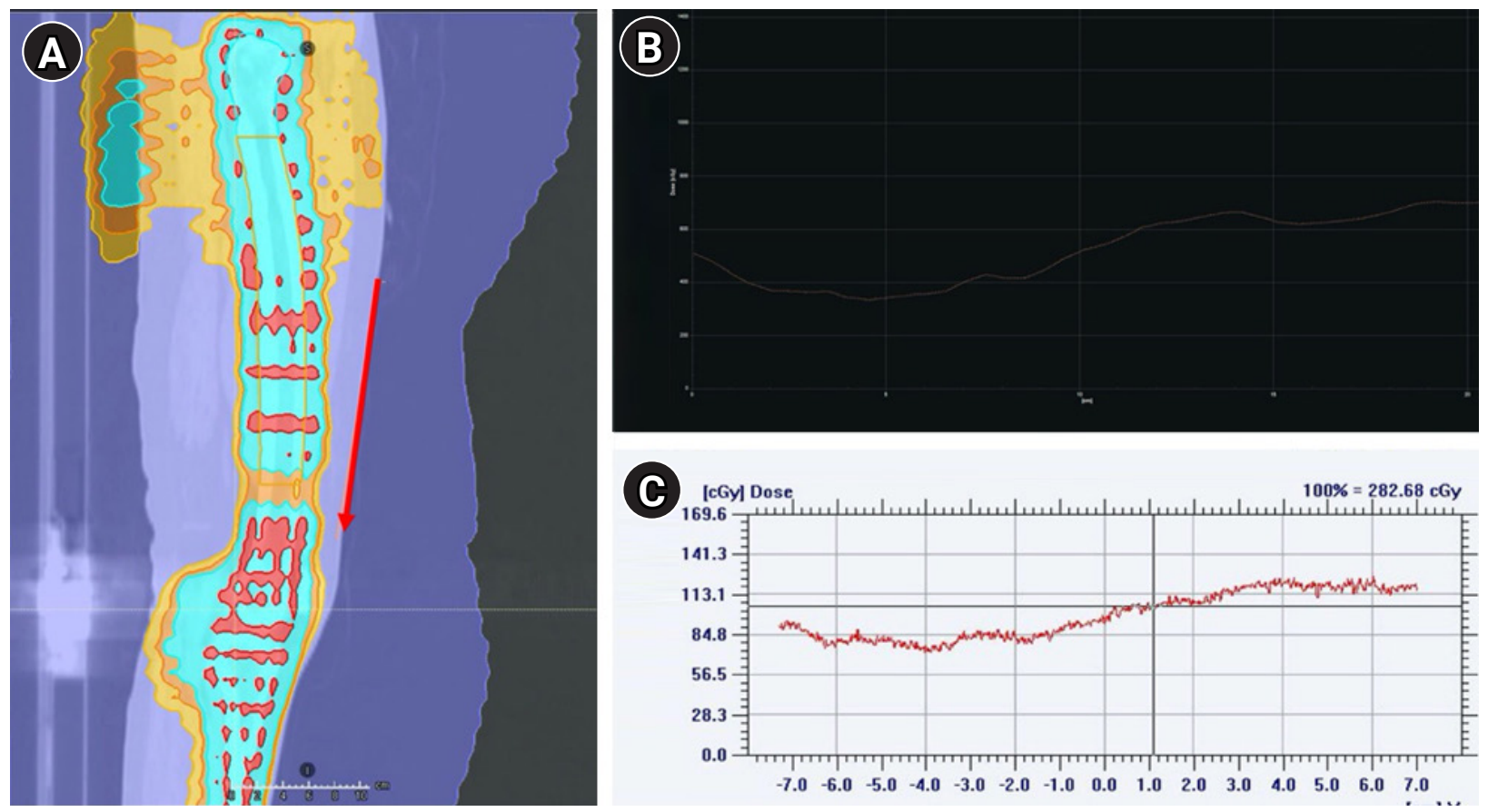

Fig. 8. In vivo junction dosimetry in a patient. (A) Sagittal view of planning computed tomography with a red arrow indicating where the film was placed on the patient for dose measurement and the region where dose profile was obtained in treatment planning system (TPS). (B) TPS measured dose profile over the specified region. (C) Dose profile obtained from Gaf-choromic film measurement over the specified region.

(range, 2.5 to 8 months)

\section{Discussion and Conclusion}

After a considerable experience with extended SSD TBI for last several years, we evaluated this methodology of TMLI, with an aim to eventually study the feasibility and safety of dose escalation in se- lected patients. Our objective with this study was to standardize the procedure, plan resources and time requirements and analyze our early experience with respect to dosimetry and treatment delivery.

We have demonstrated that the methodology adopted by us was safe and feasible. Certain complexities in our methodology were due to resource availability limitations. The major complexity in our 
workflow was using two different planning systems to complete the treatment planning and plan evaluation. This step could be simplified by using a single planning system for the entire procedure. Another challenge in our workflow was to limit patient inroom time and hence we have standardized our image guided treatment delivery protocol to minimize repeated patient re-positioning and ensure optimal treatment efficiency.

Shinde et al. [9] compared the late toxicities with TMI/TMLI with that of TBI based on historical controls. The findings in that study showed very low incidence of radiation pneumonitis, radiation related renal toxicity, hypothyroidism, and cataract formation with $\mathrm{TMI} / \mathrm{TMLI}$ compared to the historical TBI controls. The dose rate for treatment delivery in TMI/TMLI cohort was $200 \mathrm{cGy} / \mathrm{min}$ in this study (10 Gy/min in our study) which is significantly higher compared to conventional TBI dose rates which range between 5-30 $\mathrm{cGy} / \mathrm{min}$. Although, effect of dose rate on toxicities have been noted in 1-5 cGy/min range and with single fraction, there is very little evidence of its effect on toxicity with fractionated treatments even at higher dose rates. The low incidence of late toxicities as demonstrated by Shinde et al. [9] suggest that that the higher dose rate may not measurably contribute to organ dysfunction especially in the context of significant dosimetric sparing.

Helical TomoTherapy for magna-field irradiation offers a high degree of conformity, homogeneity of target coverage dose and significant sparing of OAR with optimal treatment efficiency, without the need for multiple junctions. This is possibly the reason why several groups have extensively evaluated it for TBI and TMI/TMLI [8,10-15]. Recently volumetric modulated arc therapy (VMAT) has also been evaluated in this setting to a reasonable success [16].

Although we have used helical TomoTherapy to treat our patients, the same principles can be broadly applied for VMAT as well. The target delineation for both TMI and TMLI has been varied in literature. Exclusion of mandible, maxilla, bones in the forearm, small bones of the hands and feet from the target has been diverse [1-5]. We have included the entire skeletal system excluding mandible, hyoid, laryngeal cartilage and patella.

Similarly inclusion of nodal groups varied with some authors not explicitly mentioning the nodal groups that are intentionally irradiated $[8,10-11]$. We have included cervical, mediastinal, para-aortic, pelvic and inguinal nodal chains and have excluded Waldeyer's ring and porta-hepatic nodal groups in order to limit the doses to oropharyngeal mucosa and liver/bowel respectively. There is also a discrepancy when it came to inclusion of liver, which was part of the target in some and was an OAR in a few series $[8,10,11,17]$. Patients will be followed up to validate the appropriateness of our approach.

The dose coverage and homogeneity met our pre-specified dose constraints except in upper limbs where the inhomogeneity exceeded our thresholds. This is expected with helical TomoTherapy delivery for peripherally located targets due to increased thread effect and limited beamlet views for this region.

Lung $D_{50}$ achieved in our patients was relatively higher compared to other published series of TMLI $[8,10,11,17]$ and this is possibly due to differences in target delineation, margins and differences in target coverage goals. Varying margins to the chest wall target have been used by investigators which were based either on geometric expansion ranging from no margin up to $10 \mathrm{~mm}[10,18]$ or based on chest wall motion [18]. Our coverage goal of $95 \%$ of dose to $98 \%$ of CTV was higher than the $85 \%$ set by Wong et al. [10] possibly explaining higher lung $D_{50}$. However, lung $D_{50}$ achieved for TMLI in our cohort was comparable to that achieved by Haraldsson et al. [18] for TMI. We intend to aggressively limit lung $D_{50}$ in future, especially when we escalate the doses either by using tighter margins or by relaxing the target coverage goals in the upper chest (our current mean CTV chest $\mathrm{D}_{98}$ was $99.6 \%$ ).

We have used MVCT alone as a guide for patient setup verification, which mandated diligent patient immobilization and systematic imaging protocol to minimize patient repositioning. The first MVCT (lower neck to upper abdomen) in our imaging protocol was done to manually correct any longitudinal and yaw errors before proceeding with the rest of the procedure. This step significantly reduced the magnitude of couch corrections and the need for further repositioning. Considering the large diversity in methods used for immobilization and image verification, a multi-institutional effort [19] to analyze the dosimetric precision in TMI revealed that the image verification performed using a whole body MVCT yielded lesser setup corrections and better dosimetric precision compared to partial body imaging. In our study the MVCT covered the entire body, however the imaging and treatment delivery was done in segments. Because of the relatively large PTV margins used and the minimal setup corrections achieved in our study, significant dose perturbations within the target are unlikely. We also had performed manual verification of the setup by overlaying the isodose wash on the MVCT to ensure PTV coverage by at least 95\% isodose-wash before treatment delivery for each segment.

Our patient setup errors and corrections were comparable to the one published recently by Mancosu et al. [20] which compared two different immobilization systems (baseplates with and without inter-fixation). The study showed lesser setup corrections when the baseplates were inter-fixed. We have used a whole-body vacuum bag and hence did not require any inter-fixation. In our study, special attention was given to extremities and scrotal position during immobilization and daily setup to minimize the repeated image verifications. The robustness of the dose at the junction was reaf- 
firmed with the help of film dosimetry.

As compared to TBI, TMLI is a time and resource-intensive procedure. We observed that for one patient to be successfully treated, the department would have to allocate nearly 27 physicist-hours, 21.5 therapist-hours, and 16.25 radiation oncologist-hours (Table 1). Significantly higher person-hours and in-room time as compared to other magna-field irradiations can potentially lead to stress on work-timings for all the personnel involved especially in busy radiation oncology departments. The mean in-room time of 9.25 hours per patient over 3 days, could potentially lead to significant changes in treatment schedules for other patients. We chose weekends and public holidays to treat our patients to minimize interruptions to the department's routine.

Our beam-on time was higher compared to that reported for TMI using helical TomoTherapy and VMAT [16], but comparable to others for TMLI using helical TomoTherapy $[8,10,11,17]$. Our time for target delineation, treatment planning and quality assurance was comparable to that reported by others for TBI [21]. We believe that further optimization of total in-room time can be achieved with growing experience.

Since the aim of the study was to standardize our methodology and plan resource requirements, we chose to publish this report after treating the initial 5 patients. However, the dosimetric data is limited due to the small sample size and hence

we may have to optimize the target and OAR dose constraints in the future on a case to case basis. All our patients were young and had healthy lung volumes enabling us to achieve desirable lung $D_{50}$, however this could be challenging in patients with poor lung volumes. The robust setup and minimal couch corrections noted in our study may not be possible to achieve in more challenging patients and hence may either require a more liberal margin recipe or a compromise on dose constraints. Our average in-room time of 9.25 hours per patient may pose a significant challenge while treating pediatric patients, although the need for junction would not arise in such patients $(<135 \mathrm{~cm})$.

In conclusion, TMLI results in significant sparing of OAR and provides an opportunity for safe dose escalation despite being a time and resource-intensive procedure. Our comprehensive report on the detailed methodology, resource and time requirements to implement TMLI would help departments planning to commission this procedure and plan their resources. We will be using this methodology in a prospective phase II trial to study the safety and feasibility of dose escalated TMLI as part of the conditioning regimen before ABMT.

\section{Conflict of Interest}

No potential conflict of interest relevant to this article was reported.

\section{References}

1. Shueng PW, Lin SC, Chong NS, et al. Total marrow irradiation with helical tomotherapy for bone marrow transplantation of multiple myeloma: first experience in Asia. Technol Cancer Res Treat 2009;8:29-38.

2. Cherpak AJ, Monajemi T, Chytyk-Praznik K, Mulroy L. Energy-dependent OAR sparing and dose conformity for total marrow irradiation of obese patients. J Appl Clin Med Phys 2018;19:532-8.

3. Corvo R, Zeverino M, Vagge $S$, et al. Helical tomotherapy targeting total bone marrow after total body irradiation for patients with relapsed acute leukemia undergoing an allogeneic stem cell transplant. Radiother Oncol 2011;98:382-6.

4. Lin SC, Hsieh PY, Shueng PW, Tien HJ, Wang LY, Hsieh CH. Total marrow irradiation as part of autologous stem cell transplantation for Asian patients with multiple myeloma. Biomed Res Int 2013;2013:321762.

5. Jiang B, Dai J, Zhang Y, Zhang K. Feasibility study of a novel rotational and translational method for linac-based intensity modulated total marrow irradiation. Technol Cancer Res Treat 2012; $11: 237-47$.

6. Somlo G, Spielberger R, Frankel P, et al. Total marrow irradiation: a new ablative regimen as part of tandem autologous stem cell transplantation for patients with multiple myeloma. Clin Cancer Res 2011;17:174-82.

7. ICH Good Clinical Practice. US Clinical Trials Registry [Internet]. Geneva, Switzerland: International Council for Harmonisation of Technical Requirements for Pharmaceuticals for Human Use; 2020 [cited 2020 Sep 19]. Available from: https://ichgcp.net/ clinical-trials-registryl.

8. Wong JY, Forman S, Somlo G, et al. Dose escalation of total marrow irradiation with concurrent chemotherapy in patients with advanced acute leukemia undergoing allogeneic hematopoietic cell transplantation. Int J Radiat Oncol Biol Phys 2013;85:14856.

9. Shinde A, Yang D, Frankel P, et al. Radiation-related toxicities using organ sparing total marrow irradiation transplant conditioning regimens. Int J Radiat Oncol Biol Phys 2019;105:1025-33.

10. Wong JY, Rosenthal J, Liu A, Schultheiss T, Forman S, Somlo G. Image-guided total-marrow irradiation using helical tomotherapy in patients with multiple myeloma and acute leukemia undergoing hematopoietic cell transplantation. Int J Radiat Oncol Biol 
Phys 2009;73:273-9.

11. Rosenthal J, Wong J, Stein A, et al. Phase 1/2 trial of total marrow and lymph node irradiation to augment reduced-intensity transplantation for advanced hematologic malignancies. Blood 2011;117:309-15.

12. Penagaricano JA, Chao M, Van Rhee F, Moros EG, Corry PM, Ratanatharathorn V. Clinical feasibility of TBI with helical tomotherapy. Bone Marrow Transplant 2011;46:929-35.

13. Gruen A, Ebell W, Wlodarczyk W, et al. Total body irradiation (TBI) using helical tomotherapy in children and young adults undergoing stem cell transplantation. Radiat Oncol 2013;8:92.

14. Hong CS, Kim MJ, Kim J, et al. Feasibility of hybrid TomoHelicaland TomoDirect-based volumetric gradient matching technique for total body irradiation. Radiat Oncol 2019;14:233.

15. Wilhelm-Buchstab T, Leitzen C, Schmeel LC, et al. Total body irradiation: significant dose sparing of lung tissue achievable by helical tomotherapy. Z Med Phys 2020;30:17-23.

16. Han C, Schultheisss TE, Wong JY. Dosimetric study of volumetric modulated arc therapy fields for total marrow irradiation. Radiother Oncol 2012;102:315-20.
17. Schultheiss TE, Wong J, Liu A, Olivera G, Somlo G. Image-guided total marrow and total lymphatic irradiation using helical tomotherapy. Int J Radiat Oncol Biol Phys 2007;67:1259-67.

18. Haraldsson A, Engellau J, Lenhoff S, Engelholm S, Back S, Engstrom PE. Implementing safe and robust total marrow irradiation using helical tomotherapy: a practical guide. Phys Med 2019; 60:162-7.

19. Zuro D, Vagge S, Broggi S, et al. Multi-institutional evaluation of MVCT guided patient registration and dosimetric precision in total marrow irradiation: a global health initiative by the international consortium of total marrow irradiation. Radiother Oncol 2019;141:275-82.

20. Mancosu P, Navarria P, Muren LP, Castagna L, Reggiori G, Clerici $E_{\text {, }}$ Sarina B, Bramanti S, De Philippis C, Tomatis S, Santoro A, Scorsetti M. Development of an Immobilization Device for Total Marrow Irradiation. Pract Radiat Oncol 2020;Mar 8 [Epub]. http://doi. org/10.1016/j.prro.2020.02.012.

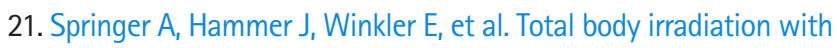
volumetric modulated arc therapy: dosimetric data and first clinical experience. Radiat Oncol 2016;11:46. 